\title{
Limited role of DWI with apparent diffusion coefficient mapping in breast lesions presenting as non-mass enhancement on dynamic contrast-enhanced MRI
}

Daly Avendano ${ }^{1,2+}$, Maria Adele Marino ${ }^{1,3+}$, Doris Leithner ${ }^{1,4}$, Sunitha Thakur ${ }^{5}$, Blanca Bernard-Davila', Danny F. Martinez ${ }^{1}$, Thomas H. Helbich ${ }^{6}$, Elizabeth A. Morris ${ }^{1}$, Maxine S. Jochelson' ${ }^{1}$, Pascal A. T. Baltzer ${ }^{6}$, Paola Clauser ${ }^{6}$, Panagiotis Kapetas ${ }^{6}$ and Katja Pinker ${ }^{1,6^{*}}$ (D)

\begin{abstract}
Background: Available data proving the value of DWI for breast cancer diagnosis is mainly for enhancing masses; DWI may be less sensitive and specific in non-mass enhancement (NME) lesions. The objective of this study was to assess the diagnostic accuracy of DWI using different ROI measurement approaches and ADC metrics in breast lesions presenting as NME lesions on dynamic contrast-enhanced (DCE) MRI.

Methods: In this retrospective study, 95 patients who underwent multiparametric MRI with DCE and DWI from September 2007 to July 2013 and who were diagnosed with a suspicious NME (BI-RADS 4/5) were included. Twenty-nine patients were excluded for lesion non-visibility on DWI ( $n=24: 12$ benign and 12 malignant) and poor DWI quality ( $n=5: 1$ benign and 4 malignant). Two readers independently assessed DWI and DCE-MRI findings in two separate randomized readings using different $A D C$ metrics and ROI approaches. NME lesions were classified as either benign $\left(>1.3 \times 10^{-3} \mathrm{~mm}^{2} / \mathrm{s}\right)$ or malignant $\left(\leq 1.3 \times 10^{-3} \mathrm{~mm}^{2} / \mathrm{s}\right)$. Histopathology was the standard of reference. ROC curves were plotted, and AUCs were determined. Concordance correlation coefficient (CCC) was measured.

Results: There were 39 malignant (59\%) and 27 benign (41\%) lesions in 66 (65 women, 1 man) patients (mean age, 51.8 years). The mean ADC value of the darkest part of the tumor (Dptu) achieved the highest diagnostic accuracy, with AUCs of up to 0.71. Inter-reader agreement was highest with Dptu ADC max (CCC 0.42) and lowest with the point tumor (Ptu) ADC min (CCC $=-0.01)$. Intra-reader agreement was highest with Wtu ADC mean $(C C C=0.44$ for reader 1, 0.41 for reader 2), but this was not associated with the highest diagnostic accuracy.
\end{abstract}

Conclusions: Diagnostic accuracy of DWI with ADC mapping is limited in NME lesions. Thirty-one percent of lesions presenting as NME on DCE-MRI could not be evaluated with DWI, and therefore, DCE-MRI remains indispensable. Best results were achieved using Dptu 2D ROI measurement and ADC mean.

Keywords: Breast cancer, Magnetic resonance imaging, Non-mass enhancement, Diffusion-weighted imaging

\footnotetext{
*Correspondence: pinkerk@mskcc.org

${ }^{\dagger}$ Daly Avendano and Maria Adele Marino contributed equally to this work. 'Department of Radiology, Breast Imaging Service, Memorial Sloan Kettering Cancer Center, Suite 705, 300 E 66th Street, New York, NY 10065, USA

${ }^{6}$ Division of Molecular and Gender Imaging, Department of Biomedical Imaging and Image-Guided Therapy, Medical University of Vienna, Vienna, Austria

Full list of author information is available at the end of the article
}

(c) The Author(s). 2019 Open Access This article is distributed under the terms of the Creative Commons Attribution 4.0 International License (http://creativecommons.org/licenses/by/4.0/), which permits unrestricted use, distribution, and reproduction in any medium, provided you give appropriate credit to the original author(s) and the source, provide a link to the Creative Commons license, and indicate if changes were made. The Creative Commons Public Domain Dedication waiver (http://creativecommons.org/publicdomain/zero/1.0/) applies to the data made available in this article, unless otherwise stated. 


\section{Background}

Although dynamic contrast-enhanced magnetic resonance imaging (DCE-MRI) of the breast is the most sensitive method for the detection of breast cancer, it is limited in assessing the likelihood of malignancy for non-mass enhancement (NME) breast lesions [1-5], resulting in unnecessary breast biopsies [6-9]. With advances in imaging techniques and hardware, such as better gradient systems and multichannel coils, DWI with ADC mapping has emerged as the most robust and reliable adjunct to DCE-MR with reported sensitivities of up to $96 \%$ and specificities of up to $100 \%$ for their combination [4, 10-13]. In addition, with recent concerns about the safety of gadolinium-containing contrast agents [14], DWI with ADC mapping has been suggested as an alternative unenhanced technique for breast cancer screening and diagnosis [15-19]. However, the majority of the available data for DWI is for enhancing masses, and concerns remain that DWI may be less sensitive and specific in the assessment of NME lesions [20]. Additionally, while it has been shown that a 2D region of interest (ROI) ADC measurement approach in the enhancing tumor with the visually assessed lowest $\mathrm{ADC}$ is the most practical and diagnostically accurate measurement in mass lesions [21-23], the best and most reliable measurement in NME lesions remains unclear. To close these gaps in knowledge, the aim of this study was to assess the diagnostic accuracy of DWI using different ROI measurement approaches and ADC metrics in breast lesions presenting as NME lesions on DCEMRI and to assess inter-reader agreement and repeatability of ADC measurements.

\section{Methods}

The local institutional review board approved this prospective single-institution study (EK 510/2009) and retrospective data analysis. The research was performed in accordance with relevant guidelines/regulations, and informed consent was obtained from all patients prior to multiparametric MRI of the breast.

\section{Patients}

A prospectively and consecutively populated research database was searched for patients who underwent multiparametric MRI of the breast with DCE and DWI between September 2007 and July 2013 and who fulfilled the following inclusion criteria: 18 years or older; not pregnant; not breastfeeding; no previous breast cancer treatment; presence of NME (BI-RADS 4-5) on DCEMRI suspicious according to BI-RADS lexicon, i.e., unilateral with segmental, focal, or linear distribution; and no contraindications for MRI or MRI contrast agents. Patients underwent breast MRI to evaluate the following conditions: (a) equivocal findings on conventional imaging (BIRADS 0), (b) suspicious lesions or lesions highly suggestive of malignancy on conventional imaging (BIRADS 4 and 5), and (c) preoperative staging of biopsy-proven breast cancer (BI-RADS 6).

We identified 95 patients who fulfilled these criteria. Of these, 29 patients were excluded for the following reasons: (a) lesion not visible on DWI and ADC map ( $n=24: 12$ benign and 12 malignant) and (b) poor DWI quality ( $n=5: 1$ benign and 4 malignant). Among the lesions that were not visible on DWI, there were $8 / 12$ ductal carcinomas in situ (DCIS), 3/12 invasive lobular carcinomas (ILC), and 1/12 invasive ductal carcinomas (IDC). Among the lesions with poor DWI quality, there were $2 / 4$ DCIS and 2/4 IDC.

Therefore, 66 patients were included for analysis. Patient selection is detailed in Fig. 1. Electronic medical records were reviewed to record patient age as well as histopathology results which included tumor grade, subtype, and receptor status for malignant lesions.

A number of patients included in this study have been analyzed and reported before in a different context [6]. In the prior study, the authors developed a BI-RADS ${ }^{\oplus}$ adapted reading for multiparametric MRI of the breast using DCE-MRI and DWI that adapted ADC thresholds to the assigned BI-RADS ${ }^{ø}$ classification and assessed the diagnostic value of this $\mathrm{BI}-\mathrm{RADS}^{\circ}$-adapted reading by an objective comparison with previously published assessment methods in patients with both mass $(n=255)$ and NME $(n=36)$ breast lesions.

The current study includes a larger patient number and focuses solely on the diagnostic value of DWI in NME lesions, including the 36 NME breast lesions that have been reported before.

\section{Magnetic resonance imaging technique}

All patients underwent 3 T MRI (Tim Trio, Siemens, Erlangen, Germany) in the prone position using a 4-channel breast coil (In Vivo, Orlando, FL, USA). In premenopausal women, MRI was performed in the second week of the menstrual cycle. Details on the MRI protocol have been previously published [24]. The DWI protocol included axial three-acquisition trace diffusion-weighted, doublerefocused, single-shot echo-planar imaging with inversion recovery fat suppression (TR/TE/TI 13,700/83/220 ms; FOV $340 \times 117 \mathrm{~mm}$; 40 slices at $3.5 \mathrm{~mm}$; matrix $192 \times 64$ (50\% oversampling); two $b$ values of 50 and $850 \mathrm{~s} / \mathrm{mm}^{2}$; bandwidth $1446 \mathrm{~Hz} /$ pixel; 3:19 $\mathrm{min}$ ).

\section{Data analysis}

Two breast radiologists (DA, MAM), each with more than 3 years of experience in interpretation of breast MRI, independently evaluated the DW images and corresponding $\mathrm{ADC}$ maps. Both readers were aware that patients had a breast lesion, but they were not provided 


\section{Patients with previously reported NME}

BI-RADS 4,5 and 6

\section{Inclusion Criteria \\ $\cdot \geq 18$ yo \\ - No pregnancy or breastfeeding}
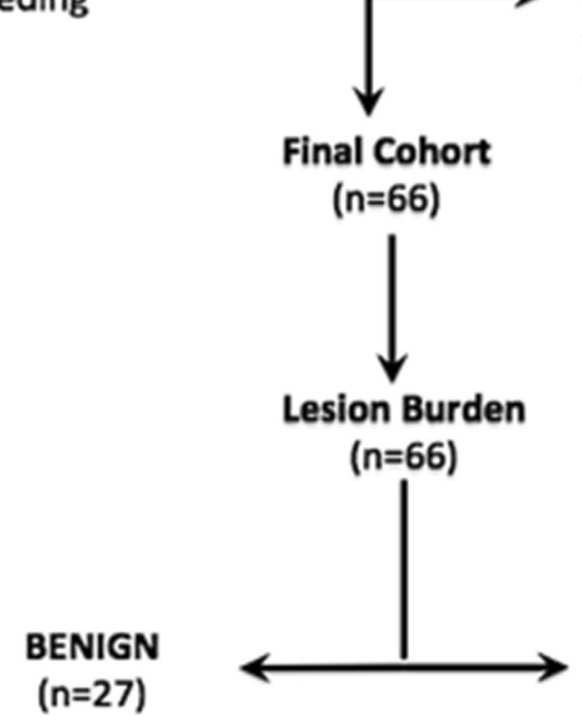

MALIGNANT

$(n=39)$

Fig. 1 The scheme summarizes the data selection criteria of our study inclusion and exclusion criteria. BI-RADS, Breast Imaging Reporting and Data System; DCIS, ductal carcinoma in situ; DWI, diffusion-weighted imaging; IDC, invasive ductal carcinoma; ILC, invasive lobular carcinoma; NME: non-mass enhancement

with previous imaging or histopathological results. Each reader performed all the readings twice, with a washout period of at least 3 weeks.

All Digital Imaging and Communications in Medicine (DICOM) images were loaded onto the open-source image processing tool OsIRIX (OsiriX Foundation, Geneva, Switzerland). The readers evaluated the lesions on both DW images and ADC maps and then recorded mean $\mathrm{ADC}$ values on $\mathrm{ADC}$ maps using three measurement approaches: (a) whole tumor (Wtu) ROI, (b) darkest part (Dptu) tumor $10 \mathrm{~mm} \mathrm{ROI,} \mathrm{and} \mathrm{(c)} \mathrm{point} \mathrm{tumor} \mathrm{(Ptu)} 3$ $\mathrm{mm}$ ROI. For Wtu delineation, the readers segmented the entire $3 \mathrm{D}$ volume of the lesion by contouring the borders for each slice. For Dptu delineation, the readers used a 10$\mathrm{mm} 2 \mathrm{D} R O I$ in the visually darkest (i.e., most suspicious) region of the enhancing tumor [9]. A similar approach was used for Ptu delineation by placing a 2D ROI point tool on the darkest part of the lesion (Fig. 2).

The mean, minimum, and maximum ADC values were recorded for Wtu and Dptu while only one ADC value was obtained for the Ptu.

A threshold of $1.3 \times 10^{-3} \mathrm{~mm}^{2} / \mathrm{s}$ for the $\mathrm{ADC}$ value was used as the cutoff for the differentiation between benign $\left(>1.3 \times 10^{-3} \mathrm{~mm}^{2} / \mathrm{s}\right)$ and malignant $\left(\leq 1.3 \times 10^{-3}\right.$ $\mathrm{mm}^{2} / \mathrm{s}$ ) lesions $[11,25,26]$.

\section{Histopathology}

Histopathological diagnosis was established using imageguided needle biopsy or surgery no later than 1 week after MRI. In the case of a benign diagnosis at image-guided needle biopsy, the final diagnosis was benign. In the case of a high-risk lesion with uncertain potential for malignancy, the final diagnosis was established with surgery.

\section{Statistical analysis}

All calculations were performed on a per-lesion basis. Univariate analysis was performed. Differences in imaging features between malignant and benign lesions were assessed using the Wilcoxon signed-rank test. ROC curves were plotted, and the area under the curve (AUC) was determined. $p$ values of $\leq 0.5$ were considered significant.

To assess intra- and inter-reader agreement, concordance correlation coefficient (CCC) was used on the continuous measures (ADC values) obtained by the two independent readers. The CCC provides a measure of both precision and accuracy in relation to the line of perfect concordance $\left(45^{\circ}\right.$ 


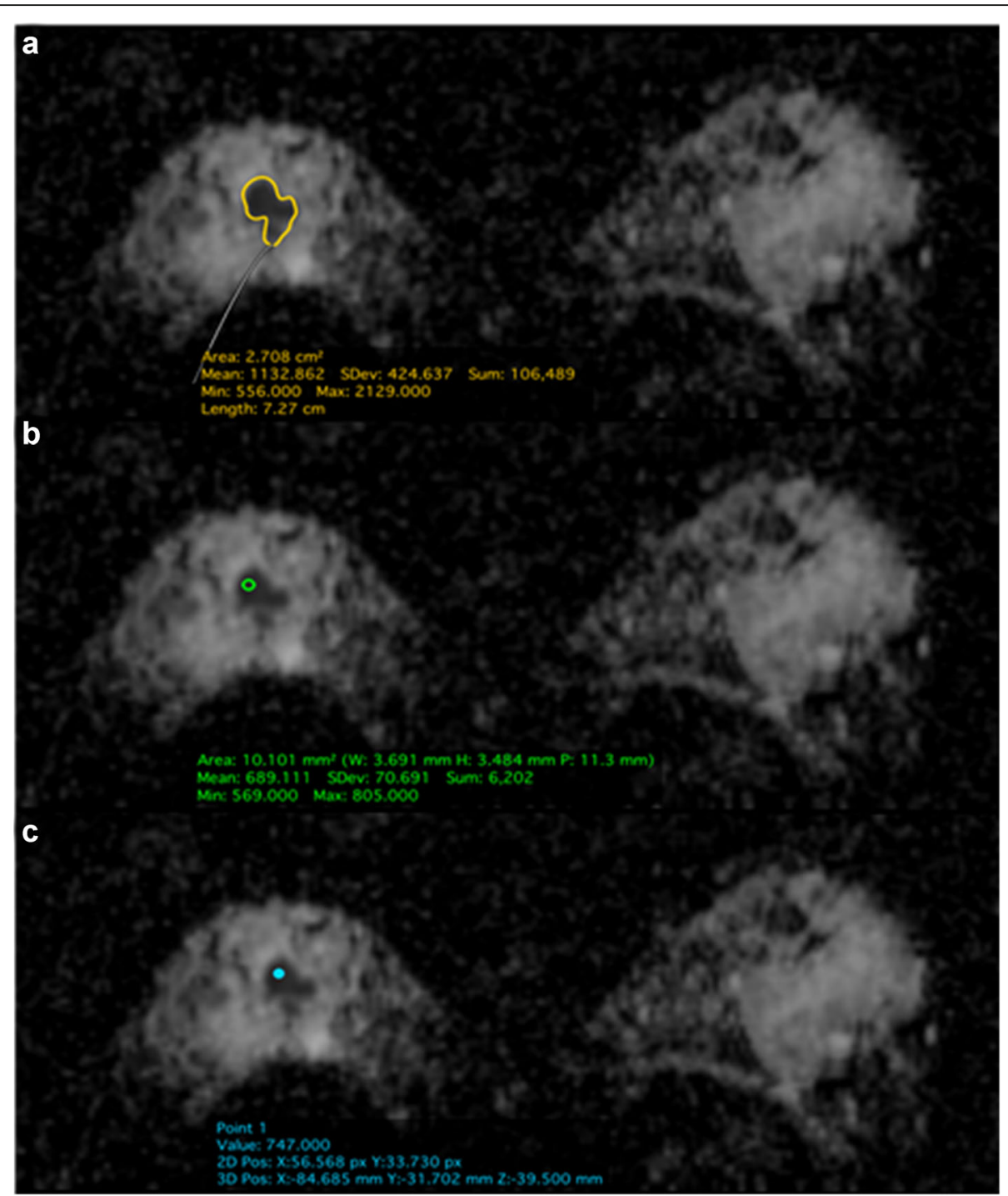

Fig. 2 Invasive ductal carcinoma, grade 2, in a 47-year-old patient undergoing preoperative MRI. Apparent diffusion coefficient (ADC), axial views $(\mathbf{a}-\mathbf{c})$. The images show a hypointense area of restricted diffusion in the central part of the right breast. Examples of the three methods used to measure the ADC values: $\mathbf{a}$ whole tumor delineation, $\mathbf{b}$ darkest part of the tumor delineation, and $\mathbf{c}$ point tumor delineation. The three regions of interest show low ADC values $<1.3 \times 10^{-3} \mathrm{~mm}^{2} / \mathrm{s}$ indicating that the enhancement is highly suspicious of malignancy (Breast Imaging Reporting and Data System 5)

line on a scatterplot). The better the agreement between the two readers for the parameter, the closer the coefficient will be to 1 . The Bland-Altman assessment was used to compare the absolute difference as a percentage of the average of reads, including 95\% limits of agreement.

\section{Results}

\section{Lesions characteristics}

There were 66 lesions in 66 patients (65 women, 1 man; mean age $51.8 \pm 10.8$ years (range 26-76 years), Table 1). Histopathology revealed 39 malignant (59\%) and 27 benign (41\%) lesions. The mean size of all lesions, as measured on DCE-MRI, was $27.8 \pm 18.3$ $\mathrm{mm}$ (range 5-80 $\mathrm{mm}$ ).

All lesions were seen on both sequences DW images and ADC map.

For a detailed description of the histopathological diagnosis and mean lesion size, see Table 2.

\section{Differentiation of benign and malignant breast tumors}

Results show that the diagnostic accuracy of DWI with ADC mapping is limited in lesions presenting as NME lesions on DCE-MRI regardless of the ROI measurement approach and different ADC metrics used. The Dptu 
Table 1 Baseline characteristics of study population

\begin{tabular}{lllll}
\hline & $N(\%)$ & Mean age (years) & Standard deviation (SD) & Range (years) \\
\hline Total of the study population & $66(100)$ & 51.8 & \pm 10.8 years & $26-76$ \\
Women & $65(98.5)$ & 51 & \pm 11.1 years & - \\
Men & $1(1.5)$ & 67 & -76 & $26-49$ \\
Premenopausal women & $32(49)$ & 42 & \pm 6 years & $50-76$ \\
Postmenopausal women & $33(51)$ & 60 & \pm 7.5 years & \\
\hline
\end{tabular}

ADC mean measurement approach most consistently showed differences in the ADC values of benign and malignant with the best AUC of 0.71 (Fig. 3).

Several ROI placement approaches were found to yield lower ADC values for malignant NME breast lesions than for benign NME breast lesions for at least one reader in one reading: Wtu ADC max (reading 1, reader 1, $p=0.01$ ), Dptu ADC max (reading 2, reader $1, p=0.08$ ), Wtu ADC mean (reading 2, reader 2, $p=0.02$ ), Wtu ADC min (reading 2, reader 2, $p=0.03$ ), Dptu $\mathrm{ADC}$ min (reading 2, reader 2, $p=$ 0.02 ), and Ptu ADC mean (reading 2 , reader $2, p=0.001$ ).

$\mathrm{ADC}$ values for all lesions and $p$ values for the three different ROI placement approaches, stratified by benign and malignant histopathological diagnosis, are shown in Table 3. The AUC derived from both readers for the differentiation between benign and malignant NME breast lesions and readings for all measurement approaches are summarized in Table 4. ROC curves are provided in Additional file 1: Figure S1, Additional file 2: Figure S2, and Additional file 3: Figure S3).
Inter- and intra-reader agreement

Inter- and intra-reader agreement in assessing ADC values was generally fair to moderate (Table 5 and Table 6). Inter-reader agreement was highest for Dptu $\mathrm{ADC} \max (\mathrm{CCC}=0.420)$ and lowest for Ptu ADC min $(C C C=-0.014)$. Although both readers achieved the best intra-reader agreement with the Wtu measurement approach $(\mathrm{CCC}=0.435$ for reader $1,0.412$ for reader 2$)$, this was not the most diagnostic accurate ADC measurement approach.

The Bland-Atman plots for measured parameters are provided in Additional file 4: Figure S4, Additional file 5: Figure S5, and Additional file 6: Figure S6, showing the percent differences of the measurements between the two readers versus the average of the two readers' measurements.

\section{Discussion}

The results of the current study show that the diagnostic accuracy of DWI with ADC mapping is limited in

Table 2 Detailed histopathological diagnosis of all malignant and benign non-mass enhancement lesions

\begin{tabular}{|c|c|c|c|c|}
\hline Histopathology & $N(\%)$ & $\begin{array}{l}\text { Mean size } \\
(\mathrm{mm})\end{array}$ & $\begin{array}{l}\text { Standard deviation } \\
(\mathrm{mm})\end{array}$ & $\begin{array}{l}\text { Range } \\
(\mathrm{mm})\end{array}$ \\
\hline Non-mass enhancement lesions & $66(100)$ & 40 & 25 & $5-98$ \\
\hline Malignant & $\begin{array}{l}39 / 66 \\
(59)\end{array}$ & 48.4 & 26 & $5-98$ \\
\hline Ductal carcinoma in situ & $4(10)$ & 33.5 & 33 & $7-89$ \\
\hline Invasive ductal carcinoma & $24(62)$ & 48 & 27 & $5-98$ \\
\hline Invasive lobular carcinoma & $9(23)$ & 56.5 & 20 & $25-85$ \\
\hline$I D C+D C I S$ & $1(2.5)$ & 30 & - & - \\
\hline $\mathrm{IDC}+\mathrm{LCIS}$ & $1(2.5)$ & 60 & - & - \\
\hline Benign & $\begin{array}{l}27 / 66 \\
(41)\end{array}$ & 28 & 18 & $5-80$ \\
\hline $\mathrm{FA} / \mathrm{FAH}$ & $5(19)$ & 21.5 & 11 & $10-38$ \\
\hline $\begin{array}{l}\text { Adenosis, sclerosing adenosis, focal fibrosis, apocrine metaplasia, breast parenchyma, } \\
\text { fibrocystic changes }\end{array}$ & $12(44)$ & 23 & 16 & $5-50$ \\
\hline Papilloma & $1(4)$ & 45 & - & - \\
\hline High-risk (CCC with atypia, papilloma with atypia) & $2(7)$ & 42 & 3 & $40-44$ \\
\hline Other (chronic abscess, gynecomastia, fat necrosis, scar tissue) & $7(26)$ & 33.5 & 24 & $6-80$ \\
\hline
\end{tabular}

IDC invasive ductal carcinoma, ILC invasive lobular carcinoma, DCIS ductal carcinoma in situ, LCIS lobular carcinoma in situ, FA fibroadenoma, FAH fibroadenomatoid hyperplasia, CCC columnar cell changes 


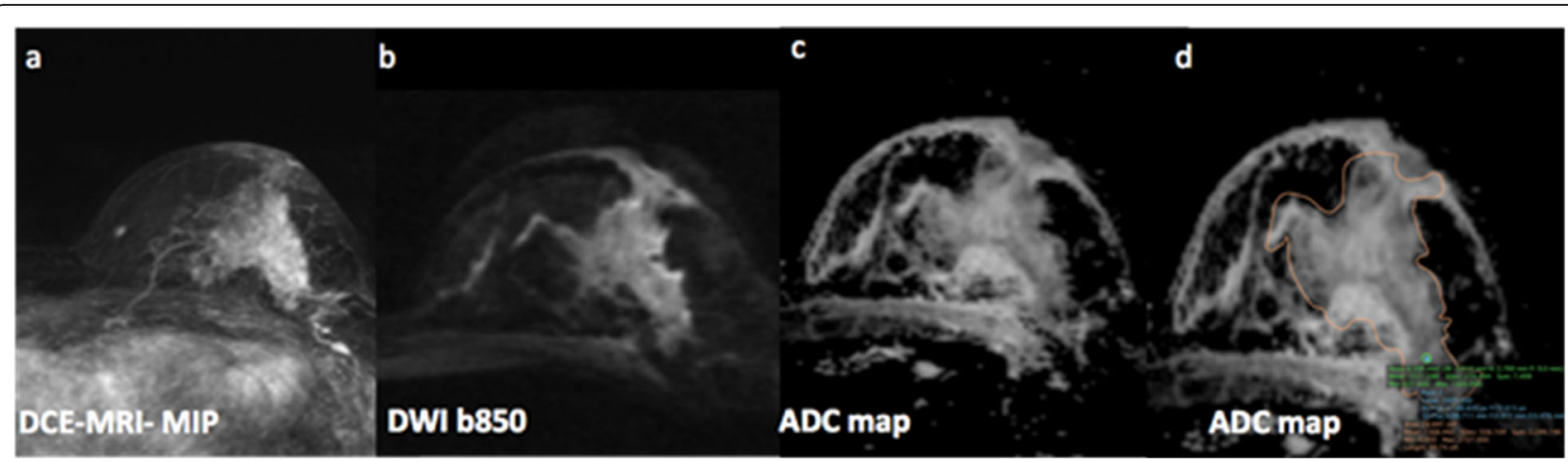

Fig. 3 Invasive lobular cancer in a 49-year-old, with biopsy-proven invasive lobular cancer, grade 2. a Dynamic contrast-enhancement MRI maximum intensity projection of the left breast shows in the upper-outer quadrant, a 63-mm segmental area of non-mass enhancement, with heterogeneous internal enhancement pattern. b Diffusion-weighted sequence at b 850 and $\mathbf{c}$ apparent diffusion coefficient (ADC) map. $\mathbf{d}$ Among the three segmentation tools, the darkest part of the tumor (Dptu) 2D region of interest (ROI) proved to yield the highest diagnostic accuracy, showing the lowest ADC values (Dptu ADC mean, $1.021 \times 10^{-3} \mathrm{~mm}^{2} / \mathrm{s}$; whole tumor $\mathrm{ROI}, 1.568 \times 10^{-3} \mathrm{~mm}^{2} / \mathrm{s}$; point tumor delineation $\left.\mathrm{ROI}, 1.029 \times 10^{-3} \mathrm{~mm}^{2} / \mathrm{s}\right)$

lesions presenting as NME lesions on DCE-MRI regardless of the ROI measurement approach and different ADC metrics used. Up to a third of NMEs cannot be evaluated with DWI, and therefore, DCE-MRI is still indispensable for detection and characterization of NME lesions.

Previous studies investigated DWI with ADC mapping for the assessment of breast lesions, mainly masses, and only few NME with reported sensitivities of up to $96 \%$ and specificities of up to $100 \%[10-13,22,27,28]$. To the best of our knowledge, this is the largest cohort of NME lesions with DCE and DWI reported so far and our results confirm the suspicion that DWI with ADC mapping performs less well for the differentiation of benign and malignant breast lesions in NME lesions than in mass lesions. Across ROI measurement approaches and different ADC metrics used in this study, the diagnostic accuracy of DWI in NME lesion is moderate at best with AUCs ranging between 0.467 and 0.736 . The Dptu ADC mean measurement approach seems to be the diagnostically yielding consistently AUCs of 0.71 . In addition, it has to be noted that in our study, 31\% (29/ 95) of lesions presenting as NME lesions on DCE-MRI could not be evaluated on DWI due to either nonvisibility ( $n=24: 12$ benign and 12 malignant) or poor DWI quality ( $n=5: 1$ benign and 4 malignant). These

Table 3 ADC values for all lesions and $p$ values for the three different ROI placement approaches

\begin{tabular}{lllllll}
\hline ADC metric $\left(\times 10^{-3} \mathrm{~mm}^{2} / \mathrm{s}\right)$ & Benign $(n=27)$ & Malignant $(n=39)$ & $\begin{array}{l}\text { Reading 1 } \\
(p \text { values })\end{array}$ & Benign $(n=27)$ & $\begin{array}{l}\text { Malignant }(n=39) \\
\text { Reading 2 }(p \\
\text { values }\end{array}$ \\
\hline Wtu ADC max (reader 1) & $2.126(0.254-2.988)$ & $2.523(198-4095)$ & $0.01^{*}$ & $2.318(1.930-3085)$ & $2.311(1.466-2.968)$ & 0.66 \\
Wtu ADC max (reader 2) & $2.395(1.881-3.568)$ & $2.563(1.620-4.095)$ & 0.12 & $2.302(1.552-3.069)$ & $2.323(1.649-2.974)$ & 0.86 \\
Wtu ADC mean (reader 1) & $1.273(0.118-1.898)$ & $1.372(0.129-2.668)$ & 0.50 & $1.392(1.004-1980)$ & $1.281(0.726-1.794)$ & 0.19 \\
Wtu ADC mean (reader 2) & $1.377(0.935-2028)$ & $1.356(0.846-2020)$ & 0.68 & $1.462(1.005-2.141)$ & $1.273(0.870-1.630)$ & 0.02 \\
Wtu ADC min (reader 1) & $0.225(0-1.671)$ & $0.195(0-1.071)$ & 1 & $0.180(0-0.731)$ & $0.187(0-1.188)$ & 0.90 \\
Wtu ADC min (reader 2) & $0.137(0-1.194)$ & $0.099(0-0.970)$ & 0.11 & $0.356(0-1.353)$ & $0.140(0-1.146)$ & $0.03^{*}$ \\
Dptu ADC max (reader 1) & $1.654(0.254-2.438)$ & $1.607(0.139-2.814)$ & 0.60 & $1.711(1.143-2.617)$ & $1.469(0-2.763)$ & $0.08^{*}$ \\
Dptu ADC max (reader 2) & $1.702(0.438-2.379)$ & $1.494(0.053-2.825)$ & 0.02 & $1.721(1.103-2.686)$ & $1.501(0.687-2.370)$ & 0.03 \\
Dptu ADC mean (reader 1) & $1.249(0.252-2.176)$ & $1.201(0.012-1.942)$ & 0.85 & $1.306(0.970-2.463)$ & $1.122(0.619-1.761)$ & $0.048^{*}$ \\
Dptu ADC mean (reader 2) & $1.996(0.303-2.009)$ & $1.154(0.044-2.363)$ & $0.06^{*}$ & $1.442(0.780-2.280)$ & $1.183(0.601-1.744)$ & $0.004^{*}$ \\
Dptu ADC min (reader 1) & $0.824(0-1.904)$ & $0.913(0-2.048)$ & 0.17 & $0.916(0-2.249)$ & $0.818(0-1.670)$ & 0.35 \\
Dptu ADC min (reader 2) & $0.871(0-2.059)$ & $0.839(0-1.994)$ & 0.75 & $1.402(0.120-9.191)$ & $0.848(0-1.476)$ & 0.02 \\
Ptu ADC mean (reader 1) & $1.127(0-1.946)$ & $1.324(0.200-6.876)$ & 0.56 & $1.238(0.470-1.963)$ & $1.144(0.540-2.174)$ & 0.19 \\
Ptu ADC mean (reader 2) & $1.512(0.281-10.005)$ & $1.018(0.284-1.942)$ & 0.206 & $1.661(0.80810 .003)$ & $1.057(0.496-1.571)$ & $0.001^{*}$ \\
\hline
\end{tabular}

$A D C$ apparent diffusion coefficient, $W t u$, whole tumor, Dptu darkest part of the tumor, Ptu point tumor

*Denotes a statistical significance 
Table 4 Area under the curve for both readers, both readings, all measurement approaches, and ADC metrics

\begin{tabular}{|c|c|c|c|c|}
\hline \multirow[t]{2}{*}{ Parameters } & \multicolumn{2}{|l|}{ Reading 1} & \multicolumn{2}{|l|}{ Reading 2} \\
\hline & $\mathrm{R} 1$ & R2 & $\mathrm{R} 1$ & R2 \\
\hline Wtu ADC max & $0.699(p=0.002)^{*}$ & $0.613(p=1.00)$ & $0.467(p=0.65)$ & $0.486(p=0.85)$ \\
\hline Wtu ADC mean & $0.549(p=0.51)$ & $0.530(p=0.67)$ & $0.596(p=0.17)$ & $0.669(p=0.01)^{*}$ \\
\hline Wtu ADCmin & $0.500(p=0.99)$ & $0.599(p=0.67)$ & $0.508(p=0.90)$ & $0.646(p=0.02)^{*}$ \\
\hline Dptu ADC max & $0.538(p=0.60)$ & $0.667(p=0.02)^{*}$ & $0.628(p=0.07)$ & $0.657(p=0.02)^{*}$ \\
\hline Dptu ADC mean & $0.512(p=0.84)$ & $0.638(p=0.05)^{*}$ & $0.644(p=0.03)^{*}$ & $0.709(p=0.001)^{*}$ \\
\hline Dptu ADC min & $0.599(p=0.17)$ & $0.523(p=0.75)$ & $0.567(p=0.36)$ & $0.669(p=0.01)$ \\
\hline Ptu ADC mean & $0.542(p=0.56)$ & $0.592(0.21)$ & $0.595(p=0.19)$ & $0.736(p=0.0002)^{*}$ \\
\hline
\end{tabular}

$A D C$ apparent diffusion coefficient, Wtu whole tumor, Dptu darkest part of the tumor, Ptu point tumor

non-visible lesions and lesions with poor DWI quality comprised not only benign entities but also 6 invasive breast cancers and 10 DCIS, highlighting that DWI alone misses breast cancer and that DCE-MRI is still indispensable for detection and characterization of NME lesions. Our findings expand on prior data in smaller NME series. Kul et al. [29] found that the effectiveness of DWI using ADC mean was lower for NME lesions as compared with masses and that the difference between the ADCs of benign and malignant lesions was smaller for NME lesions. Partridge et al. [30] reported that although DWI using a Wtu measurement approach and ADC mean is a promising tool for differential diagnosis for both masses and NME lesions, ADC measurements may be more useful for discriminating benign from malignant in masses than for discriminating lesions with NME enhancement. Recently, studies have investigated intravoxel incoherent motion and quantitative non-gaussian diffusion MRI [31-33]. It was demonstrated that higher $b$ values may be a way to improve tumor-to-tissue contrast, lesion visibility, and image quality of DWI for the detection and characterization of breast tumors. However, to date, none of these studies have specifically focused on the diagnostically challenging NME lesions and the full potential of use of higher $b$ values for improving diagnostic accuracy in NME lesions needs to be investigated in future studies.
We also aimed to answer the question of whether ADC measurements and ROI approaches other than those employed in prior studies may improve diagnostic accuracy of DWI in NME lesions. We found that the diagnostic accuracy of ADC measurements in NME lesions was significantly impacted by ROI choice and placement. Dptu ADC mean most consistently showed significant differences in ADC values of benign and malignant lesions, yielding the highest diagnostic accuracy. However, other ROI measurement approaches as well as different $\mathrm{ADC}$ metrics showed either less consistency and/or diagnostic accuracy, indicating less practicality for their clinical use. Our results are in agreement with Bickel et al. [34], who studied the influence of ROI placement and different ADC parameters on ADC values, diagnostic performance, inter-reader agreement, and measurement time in breast tumors, and who also found that ADC in NME had a lower accuracy compared with that achieved in mass lesions (AUC $=0.64-$ 0.73 vs. $0.96-0.97$ ).

To the best of our knowledge, the inter- and intrareader agreement of different ADC measurement approaches and metrics for NME lesions have not been reported. Our study shows that ADC measurements between and within readers were only slight to moderate in agreement, which is not entirely unexpected in NME lesions. The measurement approach that yielded the highest diagnostic accuracy, i.e., Dptu, achieved only

Table 5 Inter-reader agreement and concordance correlation coefficient for ADC measurements in reading 1 and reading 2

\begin{tabular}{|c|c|c|c|c|}
\hline \multirow[t]{2}{*}{ ADC metric } & \multicolumn{2}{|l|}{ Reading 1} & \multicolumn{2}{|l|}{ Reading 2} \\
\hline & CCC (95\% confidence interval) & Strength of agreement & CCC (95\% confidence interval) & Strength of agreement \\
\hline Wtu ADC max & $0.35(0.15,0.55)$ & Fair & $0.04(-0.01,0.09)$ & Slight \\
\hline Wtu ADC mean & $0.34(0.15,0.54)$ & Fair & $0.04(0.32,0.69)$ & Slight \\
\hline Wtu ADC min & $0.30(0.10,0.49)$ & Slight & $0.09(0.15,0.32)$ & Slight \\
\hline Dptu ADC max & $0.141(0.09,0.38)$ & Slight & $0.42(0.23,0.62)$ & Moderate \\
\hline Dptu ADC mean & $0.11(-0.11,0.37)$ & Slight & $0.32(0.11,0.53)$ & Fair \\
\hline Dptu ADC min & $0.13(0.03,0.19)$ & Slight & $0.03(-0.13,0.19)$ & Slight \\
\hline Ptu ADC mean & $-0.01(-0.24,0.22)$ & Slight & $0.04(-0.09,0.18)$ & Slight \\
\hline
\end{tabular}

Wtu whole tumor, Dptu darkest part of the tumor, Ptu point tumor, max maximum, min minimum 
Table 6 Intra-reader agreement and 95\% confidence intervals for reader 1 and reader 2 for all measured MRI parameters

\begin{tabular}{|c|c|c|c|c|}
\hline \multirow[t]{2}{*}{ ADC metric } & \multicolumn{2}{|l|}{ Reader 1} & \multicolumn{2}{|l|}{ Reader 2} \\
\hline & Rho-c (95\% confidence Interval) & Strength of agreement & Rho-c (95\% confidence interval) & Strength of agreement \\
\hline Wtu max ADC & $0.37(0.41,0.74)$ & Fair & $0.24(0.07,0.41)$ & Slight \\
\hline Wtu mean ADC & $0.44(0.26,0.61)$ & Moderate & $0.41(0.21,0.62)$ & Moderate \\
\hline Wtu min ADC & $0.21(-0.02,0.44)$ & Slight & $0.29(0.09,0.48)$ & Slight \\
\hline Dptu max ADC & $0.18(-0.06,0.41)$ & Slight & $0.33(0.11,0.54)$ & Fair \\
\hline Dptu mean ADC & $0.39(0.20,0.59)$ & Fair & $0.05(-0.01,0.12)$ & Slight \\
\hline Dptu min ADC & $0.37(0.41,0.74)$ & Fair & $0.24(0.07,0.41)$ & Slight \\
\hline Ptu mean ADC & $0.44(0.26,0.61)$ & Moderate & $0.41(0.21,0.62)$ & Moderate \\
\hline
\end{tabular}

$\overline{A D C}$ apparent diffusion coefficient, Wtu, whole tumor, Dptu darkest part of the tumor, Ptu point tumor, max maximum, min minimum

slight to fair inter- and intra-reader agreement, which is likely due to the placement of the ROIs after subjective radiologist's review; therefore, not necessarily the same ROI location is chosen by each reader and for each reading. Moderate intra-reader agreement was achieved with both Wtu ADC mean measurement and Ptu ADC mean, but these were not the approaches with the highest diagnostic accuracy. The moderate agreement for Wtu ADC mean was most likely because NME lesions are often difficult to delineate, and therefore, the size and shape of ROI is more prone to variation even within readers. For $\mathrm{Ptu}$, not necessarily the same point is chosen in each reading, leading to different $\mathrm{ADC}$ values. As for the intra-reader agreement, Dptu ADC max reached moderate intra-reader agreement. While the inter-reader agreement for Wtu ADC mean was more consistent between readers, it was not associated with the best diagnostic accuracy. Additionally, for the purpose of breast cancer diagnosis, a 2D ROI ADC mean measurement approach seems most practical.

Whereas this study focused solely on NME lesions, other studies have reported inter- and intra-reader agreement for DWI with ADC in lesions that included mostly masses. Bickel et al. [34] found that minimum ADC showed the best diagnostic performance (AUC 0.93-0.96), followed by mean ADC obtained from 2D ROIs (0.93-0.94), and both achieved high intra- (ICC $0.85-0.94$ ) and inter-reader reproducibility (ICC $0.74-$ 0.94). Median measurement time was significantly shorter for the 2D ROIs $(p<0.001)$. It should be noted that there were only $29 \mathrm{NME}$ lesions in this patient population. Furthermore, when considering only NME lesions, the ADC achieved in their cohort was less accurate than in ours (AUC $=0.64-0.73$ ). Spick et al. [27] led an intra-individual prospective clinical study of 40 consecutive patients with suspicious findings, including only 8 NME lesions. Reproducibility and repeatability showed high agreement for repeated examinations, readers, and measurements (all ICCs $>0.9$, coefficient of variations $3.2-8 \%$ ), indicating little variation. The Bland-Altman plots demonstrated no systematic differences, and diagnostic accuracy was not significantly different in the two repeated examinations (all ROC curves $>0.91, p>$ $0.05)$. There is consensus that reproducibility, repeatability, and diagnostic accuracy of DWI is necessary for its use as a potential quantitative imaging characteristic to enable improved breast lesion detection, characterization, and assessment of treatment response. Newitt et al. [35] evaluated the repeatability and reproducibility of breast tumor ADC in a multi-institution clinical trial setting, using standardized DWI protocols and quality assurance procedures. ADC repeatability was excellent in $80 \%(71 / 89)$ of cases. However, the authors did not report the number of NME lesions in their cohort. In contrast to other studies that reported interand intra-agreement for DWI with ADC mapping results for masses, our study demonstrated poorer reproducibility (fair to moderate). However, considering that our cohort consisted exclusively on NME lesions, this was expected.

Our study has some limitations. The sample size of our cohort is relatively small, but to the best of our knowledge, this is the largest cohort of NME lesions with DCE $(n=95)$ and DWI $(n=66,24$ not visible in DWI and 5 DWI quality insufficient) reported so far. This study was also conducted at a single tertiary center institution, and the interpretations were performed by experienced breast fellowship-trained radiologists, potentially making it difficult to extrapolate to community practice. Therefore, the overall rate of malignancy is high and the results might not be applicable to every radiologist, but it has to be noted that international guidelines [26] recommend that clinical breast MRI is reported by breast specialists. Another limitation is the retrospective nature of this study; therefore, the acquired different ADC measurements were not used in clinical decision-making. However, such retrospective studies are necessary to gather relevant information to allow future standardization and facilitate optimal clinical application implementation. Further, only BI-RADS 4 and 5 NMEs were included in the study and this could have caused a selection bias, leading to potential issues with 
statistical power, precision, and validity. Additional studies with larger cohorts are required to confirm our findings.

\section{Conclusions}

In conclusion, the accuracy of DWI with ADC mapping is limited in breast tumors presenting as NME lesions, with best results being achieved using ADC mean and a 2D ROI measurement approach. Up to a third of NMEs cannot be evaluated with DWI, and therefore, DCE-MRI remains indispensable.

\section{Supplementary information}

Supplementary information accompanies this paper at https://doi.org/10. 1186/s13058-019-1208-y.

Additional file 1: Figure S1. ROC curves and AUC (in brackets) for the minimum, mean, and maximum ADC of the Whole Tumor 3D ROI segmentation approach.

Additional file 2: Figure S2. ROC curves and AUC (in brackets) for the minimum, mean and maximum ADC of the Darkest Part of the Tumor 2D ROI segmentation approach.

Additional file 3: Figure S3. ROC curves and AUC (in brackets) for the mean ADC of the Point Tumor ROI segmentation approach.

Additional file 4: Figure S4. Scatterplots of concordance correlation coefficients between reader 1 and reader 2 at the Time 1 and at Time 2 regarding whole tumor (WTu) apparent diffusion coefficient (ADC) maximum $(A, B)$, WTu ADC mean $(C, D)$, and WTu ADC minimum $(E, F)$.

Additional file 5: Figure S5. Scatterplots of concordance correlation coefficients between reader 1 and reader 2 at the Time 1 and at Time 2 regarding darkest part of the tumor (DpTu) apparent diffusion coefficient $(A D C)$ maximum $(A, B)$, DpTu $A D C$ mean $(C, D)$, and DpTu $A D C$ minimum $(\mathrm{E}, \mathrm{F})$.

Additional file 6: Figure S6. Scatterplots of concordance correlation coefficients between reader 1 and reader 2 at Time 1 and at Time 2 regarding Point Tumor (Ptu) apparent diffusion coefficient (ADC) mean $(A, B)$.

\section{Abbreviations}

ADC: Apparent diffusion coefficient; BI-RADS: Breast Imaging Reporting and Data System; DCE: Dynamic contrast-enhanced; DWl: Diffusion-weighted imaging; MRI: Magnetic resonance imaging; NME: Non-mass enhancement; ROI: Region of interest

\section{Acknowledgements}

The authors gratefully acknowledge the support in manuscript writing and editing from Joanne Chin.

\section{Authors' contributions}

KP, MAM, and DA contributed to the study conception and design. KP, BB-D, MAM, DA, and ST contributed to the development of methodology. DA, MAM, and DL contributed to the acquisition of data. KP, MAM, DA, BB-D, and ST contributed to the analysis and interpretation of data. KP, MAM, DA, DL, ST, MSJ, PATB, PC, PK, THH, and EAM contributed to the writing, review, and/ or revision of the manuscript. EAM, DFM, and THH were involved in the administrative, technical, or material support. KP, EAM, and THH were involved in the study supervision. All authors read and approved the final manuscript.

\section{Funding}

Funding was provided by the 2020-Research and Innovation Framework Programme PHC-11-2015 no. 667211-2, the EU Horizon 2020 project no. 688188, H2020-FETOPEN-2018-2019-2020-01 \# 828978 A Body Scan for Cancer Detection using Quantum Technology (CANCERSCAN), and seed grants from Novomed, and Guerbet, France. Daly Avendano, Maria Adele Marino,
Doris Leithner, Sunitha Thakur, Blanca Bernard-Davila, Danny Martinez, Katja Pinker, Maxine Jochelson, and Elizabeth Morris were also funded in part through the $\mathrm{NIH} / \mathrm{NCI}$ Cancer Center Support Grant P30 CA008748, the Susan G. Komen Foundation, and the Breast Cancer Research Foundation.

\section{Availability of data and materials}

The datasets used and/or analyzed during the current study are available from the corresponding author on reasonable request.

\section{Ethics approval and consent to participate}

This single-institution retrospective study was compliant with Health Insurance Portability and Accountability Act guidelines and approved by the Memorial Sloan Kettering Cancer Center Institutional Review Board with a waiver of written informed consent.

\section{Consent for publication}

Not applicable

\section{Competing interests}

Daly Avendano received travel/accommodations/meeting expenses from BD (BARD) for an interventional masterclass in London. Katja Pinker received payment for activities not related to the present article including lectures including service on speakers bureaus and for travel/accommodations/ meeting expenses unrelated to activities listed from the European Society of Breast Imaging (MRI educational course, annual scientific meeting) and the IDKD 2019 (educational course). Maxine S Jochelson has received an honorarium from GE for speaking, and an honorarium for speaking at the Lynn Sage Breast Cancer Symposium and at MD Anderson. Elizabeth A Morris has received a grant from GRAIL. The other authors declare that they have no competing interests.

\section{Author details}

${ }^{1}$ Department of Radiology, Breast Imaging Service, Memorial Sloan Kettering Cancer Center, Suite 705, 300 E 66th Street, New York, NY 10065, USA. ${ }^{2}$ Department of Breast Imaging, Breast Cancer Center TecSalud, ITESM Monterrey, Monterrey, Nuevo Leon, Mexico. ${ }^{3}$ Department of Biomedical Sciences and Morphologic and Functional Imaging, University of Messina, Messina, Italy. ${ }^{4}$ Department of Diagnostic and Interventional Radiology, University Hospital Frankfurt, Frankfurt, Germany. ${ }^{5}$ Department of Medical Physics, Memorial Sloan Kettering Cancer Center, 1275 York Ave, New York, NY 10065, USA. ${ }^{6}$ Division of Molecular and Gender Imaging, Department of Biomedical Imaging and Image-Guided Therapy, Medical University of Vienna, Vienna, Austria.

Received: 10 July 2019 Accepted: 3 October 2019

Published online: 04 December 2019

\section{References}

1. Gutierrez RL, DeMartini WB, Eby PR, Kurland BF, Peacock S, Lehman CD. BIRADS lesion characteristics predict likelihood of malignancy in breast MRI for masses but not for nonmasslike enhancement. AJR Am J Roentgenol. 2009:193(4):994-1000

2. Benndorf M, Baltzer PAT, Kaiser WA. Assessing the degree of collinearity among the lesion features of the MRI BI-RADS lexicon. Eur J Radiol. 2011:80(3):e322-4.

3. Illan IA, Ramirez J, Gorriz JM, et al. Automated detection and segmentation of nonmass-enhancing breast tumors with dynamic contrast-enhanced magnetic resonance imaging. Contrast Media Mol Imaging. 2018;2018:5308517.

4. Marino MA, Clauser P, Woitek R, Wengert GJ, Kapetas P, Bernathova M, Pinker-Domenig K, Helbich TH, Preidler K, Baltzer PA. A simple scoring system for breast MRI interpretation: does it compensate for reader experience? Eur Radiol. 2016;26(8):2529-37.

5. Helbich TH. Contrast-enhanced magnetic resonance imaging of the breast. Eur J Radiol. 2000:34(3):208-19.

6. Pinker $\mathrm{K}$, Bickel $\mathrm{H}$, Helbich $\mathrm{TH}$, et al. Combined contrast-enhanced magnetic resonance and diffusion-weighted imaging reading adapted to the "Breast Imaging Reporting and Data System" for multiparametric 3-T imaging of breast lesions. Eur Radiol. 2013;23(7):1791-802

7. Spick C, Baltzer PAT. Diagnostic utility of second-look US for breast lesions identified at MR imaging: systematic review and meta-analysis. Radiology. 2014;273(2):401-9. 
8. Spick C, Pinker-Domenig K, Rudas M, Helbich TH, Baltzer PA. MRI-only lesions: application of diffusion-weighted imaging obviates unnecessary MR-guided breast biopsies. Eur Radiol. 2014;24(6):1204-10.

9. Kuhl CK. Current status of breast MR imaging. Part 2. Clinical applications. Radiology. 2007;244(3):672-91.

10. Baltzer PAT, Kapetas P, Marino MA, Clauser P. New diagnostic tools for breast cancer. Memo. 2017;10(3):175-80.

11. Marini C, lacconi C, Giannelli M, Cilotti A, Moretti M, Bartolozzi C. Quantitative diffusion-weighted MR imaging in the differential diagnosis of breast lesion. Eur Radiol. 2007;17(10):2646-55.

12. Guo Y, Cai Y-Q, Cai Z-L, et al. Differentiation of clinically benign and malignant breast lesions using diffusion-weighted imaging. J Magn Reson Imaging JMRI. 2002;16(2):172-8.

13. Rahbar H, Zhang Z, Chenevert TL, et al. Utility of diffusion-weighted imaging to decrease unnecessary biopsies prompted by breast MRI: a trial of the ECOG-ACRIN cancer research group (A6702). Clin Cancer Res Off J Am Assoc Cancer Res. 2019;25(6):1756-65.

14. Gulani V, Calamante F, Shellock FG, Kanal E, Reeder SB, International Society for Magnetic Resonance in Medicine. Gadolinium deposition in the brain: summary of evidence and recommendations. Lancet Neurol. 2017;16(7):564-70.

15. Leithner D, Moy L, Morris EA, Marino MA, Helbich TH, Pinker K. Abbreviated MRI of the Breast: Does It Provide Value? J Magn Reson Imaging. 2019;49(7): e85-e100.

16. Baltzer PAT, Benndorf M, Dietzel M, Gajda M, Camara O, Kaiser WA. Sensitivity and specificity of unenhanced MR mammography (DWI combined with T2-weighted TSE imaging, ueMRM) for the differentiation of mass lesions. Eur Radiol. 2010;20(5):1101-10.

17. Bickelhaupt S, Laun FB, Tesdorff J, et al. Fast and noninvasive characterization of suspicious lesions detected at breast cancer X-ray screening: capability of diffusion-weighted MR imaging with MIPs. Radiology. 2016;278(3):689-97.

18. Melsaether A, Gudi A. Breast magnetic resonance imaging performance: safety, techniques, and updates on diffusion-weighted imaging and magnetic resonance spectroscopy. Top Magn Reson Imaging TMRI. 2014;23(6):373-84.

19. Bickelhaupt $\mathrm{S}$, Tesdorff J, Laun FB, et al. Independent value of image fusion in unenhanced breast MRI using diffusion-weighted and morphological T2weighted images for lesion characterization in patients with recently detected BI-RADS 4/5 x-ray mammography findings. Eur Radiol. 2017;27(2):562-9.

20. Pinker K, Moy L, Sutton EJ, Mann RM, Weber M, Thakur SB, Jochelson MS, Bago-Horvath Z, Morris EA, Baltzer PA, Helbich TH. Diffusion-weighted imaging with apparent diffusion coefficient mapping for breast cancer detection as a stand-alone parameter: comparison with dynamic contrastenhanced and multiparametric magnetic resonance imaging. Invest Radiol. 2018;53(10):587-95.

21. Clauser P, Marcon M, Maieron M, Zuiani C, Bazzocchi M, Baltzer PA. Is there a systematic bias of apparent diffusion coefficient (ADC) measurements of the breast if measured on different workstations? An inter- and intra-reader agreement study. Eur Radiol. 2016;26(7):2291-6.

22. Arponen $\mathrm{O}$, Arponent $\mathrm{O}$, Sudah $\mathrm{M}$, et al. Diffusion-weighted imaging in 3.0 Tesla breast mri: diagnostic performance and tumor characterization using small subregions vs. whole tumor regions of interest. PloS One. 2015;10(10):e0138702

23. Lambregts DMJ, Beets GL, Maas M, et al. Tumour ADC measurements in rectal cancer: effect of $\mathrm{ROI}$ methods on $\mathrm{ADC}$ values and interobserver variability. Eur Radiol. 2011;21(12):2567-74.

24. Bogner W, Gruber S, Pinker K, et al. Diffusion-weighted MR for differentiation of breast lesions at 3.0 T: how does selection of diffusion protocols affect diagnosis? Radiology. 2009;253(2):341-51.

25. Pinker K, Marino MA, Dr Meyer-Baese A, Helbich TH. Multiparametric and molecular imaging of breast tumors with MRI and PET/MRI. Radiol. 2016;56(7):612-21.

26. Mann RM, Balleyguier C, Baltzer PA, Bick U, Colin C, Cornford E, Evans A, Fallenberg E, Forrai G, Fuchsjäger MH, Gilbert FJ, Helbich TH, HeywangKöbrunner SH, Camps-Herrero J, Kuhl CK, Martincich L, Pediconi F, Panizza P, Pina L, Pijnappel RM, Pinker-Domenig K, Skaane P, Sardanelli F; European Society of Breast Imaging (EUSOBI), with language review by Europa Donna-The European Breast Cancer Coalition. Breast MRI: EUSOBI recommendations for women's information. Eur Radiol. 2015;25(12):3669-78.

27. Spick C, Bickel H, Pinker K, et al. Diffusion-weighted MRI of breast lesions: a prospective clinical investigation of the quantitative imaging biomarker characteristics of reproducibility, repeatability, and diagnostic accuracy. NMR Biomed. 2016;29(10):1445-53.
28. An YY, Kim SH, Kang BJ. Differentiation of malignant and benign breast lesions: added value of the qualitative analysis of breast lesions on diffusionweighted imaging (DWI) using readout-segmented echo-planar imaging at 3.0 T. Plos One. 2017;12(3):e0174681.

29. Kul S, Eyuboglu I, Cansu A, Alhan E. Diagnostic efficacy of the diffusion weighted imaging in the characterization of different types of breast lesions. J Magn Reson Imaging JMRI. 2014;40(5):1158-64.

30. Partridge SC, Stone KM, Strigel RM, DeMartini WB, Peacock S, Lehman CD. Breast DCE-MRI: influence of postcontrast timing on automated lesion kinetics assessments and discrimination of benign and malignant lesions. Acad Radiol. 2014;21(9):1195-203.

31. lima M, Kataoka M, Kanao S, et al. Intravoxel incoherent motion and quantitative non-Gaussian diffusion MR imaging: evaluation of the diagnostic and prognostic value of several markers of malignant and benign breast lesions. Radiology. 2018;287(2):432-41.

32. Bickel H, Polanec SH, Wengert G, Pinker K, Bogner W, Helbich TH, Baltzer PA. Diffusion-weighted MRI of breast cancer: improved lesion visibility and image quality using synthetic b-values. J Magn Reson Imaging. 2019;50(6): 1754-61.

33. Goto M, Le Bihan D, Yoshida M, Sakai K, Yamada K. Adding a model-free diffusion MRI marker to BI-RADS assessment improves specificity for diagnosing breast lesions. Radiology. 2019;292(1):84-93.

34. Bickel $H$, Pinker $K$, Polanec $S$, et al. Diffusion-weighted imaging of breast lesions: region-of-interest placement and different ADC parameters influence apparent diffusion coefficient values. Eur Radiol. 2017;27(5):1883-92.

35. Newitt DC, Zhang Z, Gibbs JE, Partridge SC, Chenevert TL, Rosen MA, Bolan PJ, Marques HS, Aliu S, Li W, Cimino L, Joe BN, Umphrey H, Ojeda-Fournier H, Dogan B, Oh K, Abe H, Drukteinis J, Esserman LJ, Hylton NM; ACRIN Trial Team and I-SPY 2 TRIAL Investigators. Test-retest repeatability and reproducibility of ADC measures by breast DWI: Results from the ACRIN 6698 trial. J Magn Reson Imaging. 2019;49(6):1617-28.

\section{Publisher's Note}

Springer Nature remains neutral with regard to jurisdictional claims in published maps and institutional affiliations.
Ready to submit your research? Choose BMC and benefit from:

- fast, convenient online submission

- thorough peer review by experienced researchers in your field

- rapid publication on acceptance

- support for research data, including large and complex data types

- gold Open Access which fosters wider collaboration and increased citations

- maximum visibility for your research: over $100 \mathrm{M}$ website views per year

At $\mathrm{BMC}$, research is always in progress.

Learn more biomedcentral.com/submissions 